\title{
Organic single-crystal phototransistor with unique wavelength-detection characteristics
}

\author{
Mengxiao $\mathrm{Hu}^{1,2}$, Jinyu Liu ${ }^{2}$, Qiang Zhao ${ }^{2}$, Dan Liu ${ }^{1,2}$, Qing Zhang ${ }^{2}$, Ke Zhou ${ }^{2}$, Jie $\mathrm{Li}^{2}$, \\ Huanli Dong ${ }^{1,2^{*}}$ and Wenping $\mathrm{Hu}^{2,3}$
}

\begin{abstract}
Organic phototransistors based on high-quality 2,8-dichloro-5,11-dihexyl-indolo[3,2-b]carbazo (CHICZ) single crystals show the highest photoresponsivity of $3 \times 10^{3} \mathrm{~A} \mathrm{~W}^{-1}$, photosensitivity of $2 \times 10^{4}$ and the detectivity can achieve $8.4 \times 10^{14}$ Jones. We also discovered good linear dependence of $\log$ (photosensitivity) versus the wavelength when the devices were illuminated with a series of sameintensity but different-wavelength lights. The organic phototransistors based on CHICZ single crystal have potential applications in wavelength-detection.
\end{abstract}

Keywords: organic phototransistor, single crystal, photoresponsivity, photosensitivity, wavelength-detection

\section{INTRODUCTION}

Phototransistors are widely used in optoelectronic fields, such as photo-sensors, opto-isolators, image sensors, optically controlled phase shifters, optoelectronic switches, high-speed photodetectors and integrated circuits due to their properties of electric control and electric synchronization [1-4]. As the inorganic phototransistors developed rapidly, recently, the research on organic phototransistors (OPTs) becomes a hot topic [57] due to their low cost, light weight, and easy fabrication [8].

Strong absorption and efficient charge transport as well as superior photoelectric conversion are important factors for organic semiconductors $[9,10]$, which are determined by the intrinsic structure of the materials and the subsequent processing. The best charge transport and photoelectrical properties of organic semiconductors are always observed in single-crystal [11-19] due to their intrinsically long-range molecular orders, low defects and grain boundaries. To date, various OPTs based on organic semiconductor single crystals have been reported with special focus on their characteristics of high on/off ratio under light illumination and light intensity-detection ability $[1,2,8]$. However, few reports for OPTs involve the investigation on the wavelength-detection characteristics. In this paper, we constructed OPTs based on organic single crystal semiconductor of 2,8-dichloro-5,11-dihexylindolo[3,2-b]carbazo (CHICZ) [20]. The results demonstrated that CHICZ single crystal based-OPTs exhibited high photoresponsivity of $3 \times 10^{3} \mathrm{~A} \mathrm{~W}^{-1}$, photosensitivity of $2 \times 10^{4}$ and detectivity of $8.4 \times 10^{14}$ Jones. Moreover, for the first time, a good linear correlation between $\log$ (photosensitivity) versus the wavelength for CHICZbased OPTs is obtained with the linear correlation coefficient as high as 0.980 from 400 to $600 \mathrm{~nm}$ when the devices were illuminated with a series of sameintensity but different-wavelength lights. This new discovery opens a new avenue to extend the application fields of OPTs in organic optoelectronics, such as the unique wavelength-detection devices.

\section{EXPERIMENTAL SECTION}

\section{Preparation of CHICZ single crystals}

High-quality CHICZ single crystals for phototransistors were prepared by physical vapor transport (PVT) [21]. The CHICZ powder was added to a quartz boat, which was laid on a heating zone in the tube furnace, and the tube furnace was heated to about $150^{\circ} \mathrm{C}$ for $3 \mathrm{~h}$ in vacuum. Single crystals of CHICZ were deposited directly

\footnotetext{
${ }^{1}$ Beijing Key Laboratory for Optical Materials and Photonic Devices, Department of Chemistry, Capital Normal University, Beijing 100048, China ${ }^{2}$ Beijing National Laboratory for Molecular Sciences, Key Laboratory of Organic Solids, Institute of Chemistry, Chinese Academy of Sciences, Beijing 100190, China

${ }^{3}$ Department of Chemistry, School of Science, Collaborative Innovation Center of Chemical Science and Engineering, Tianjin University, Tianjin 300072, China

* Corresponding author (email: dhl522@iccas.ac.cn)
} 
a
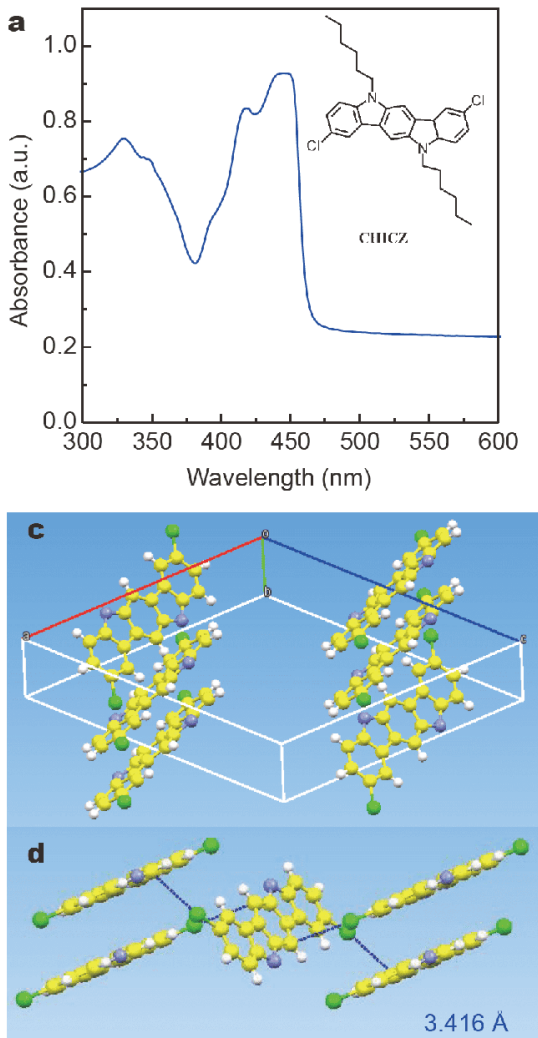
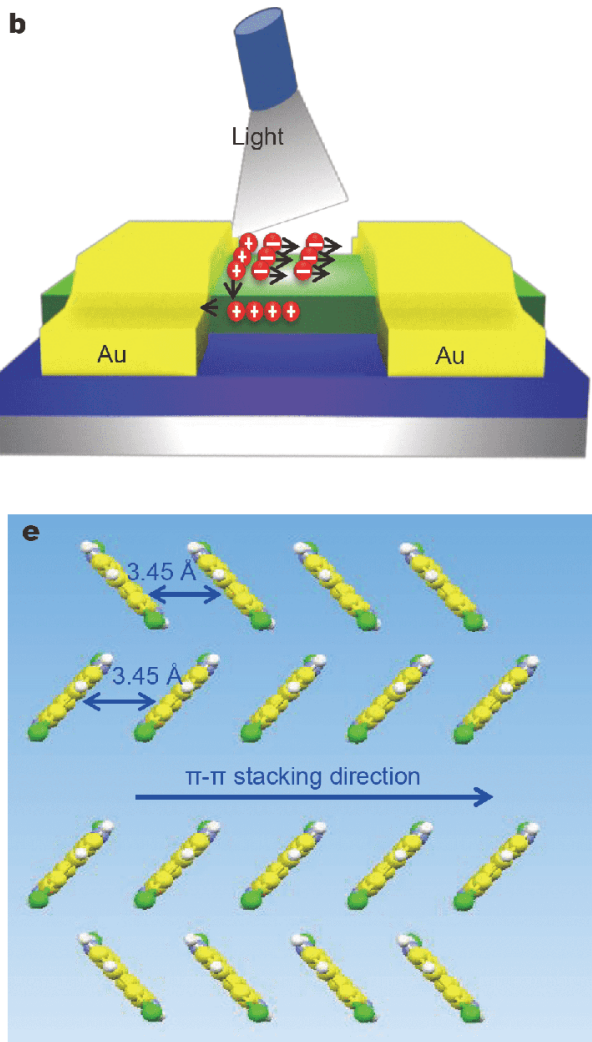

Figure 1 (a) UV-vis spectra of CHICZ crystals, inset: the molecular structure of CHICZ. (b) Schematic diagram of CHICZ single crystal phototransistor with light illumination from the top side. (c-e) Molecular packing structure of CHICZ in single crystals, seen from different directions.

on the substrate in the low temperature zone, which were subsequently used for in-situ device fabrication.

\section{Fabrication and measurement of CHICZ phototransistors} The $\mathrm{SiO}_{2}(300 \mathrm{~nm}) / \mathrm{Si}$ substrate was successively cleaned by deionized water, piranha solution (hydrogen peroxide/ concentrated sulfuric acid $(1: 2 v / v))$, pure water and isopropanol and finally dried by nitrogen flow. Then the $\mathrm{SiO}_{2}(300 \mathrm{~nm}) / \mathrm{Si}$ substrate was modified with a selfassembled monolayer of orthotrichlorosilane (OTS) to optimize the surface contact quality to enhance the devices performance.

Bottom-gate top-contacted organic field effect transistors (OFETs) based on the CHICZ crystals were constructed on the OTS-modified $\mathrm{SiO}_{2} / \mathrm{Si}$ substrates (the low resistance $\mathrm{Si}$ as gate, $300 \mathrm{~nm} \mathrm{SiO}_{2}$ as insulator with a $C=11 \mathrm{nF} \mathrm{cm}^{-2}$ ) using "organic ribbon mask" technique [22]. We covered the target single crystal with an organic ribbon, and then executed the deposition of gold, finally peeled the mask ribbon off to obtain the desired devices. Electrical and photoresponse properties were characterized by a Keithley (4200 SCS) semicon- ductor analysis instrument in ambient conditions. The photoresponsivities of different wavelengths were tested with the assistance of the xenon lighted source.

\section{RESULTS AND DISCUSSION}

Fig. 1a shows the ultraviolet-visible (UV-vis) absorption spectrum of CHICZ single crystals and the inset shows its molecular structure. Obviously, CHICZ single crystals demonstrate strong light absorption with the lowest $0-0$ transition at $446 \mathrm{~nm}$ along with the $0-1$ transitions at $418 \mathrm{~nm}$, illustrating the wide range absorption. In the $\mathrm{CHICZ}$ organic field effect transistors, the semiconductor layer was constructed on the OTS-modified $\mathrm{SiO}_{2} / \mathrm{Si}$ substrate to investigate the photoresponsivity of CHICZ. Photo-induced carriers appeared and transferred when the devices were illuminated (Fig. 1b). The CHICZ single crystal belongs to $P 2_{1} / n$ monoclinic space group with the unit cell parameters of $a=16.759(5) \AA, b=4.4914(14) \AA, c=$ 18.515(7) $\AA$ and $\beta=114.283(5)^{\circ} \quad$ [23]. Interestingly, CHICZ exhibits a coplanar structure without any molecular torsion. In a unit cell, there are four CHICZ molecules and each of them interacts with four 

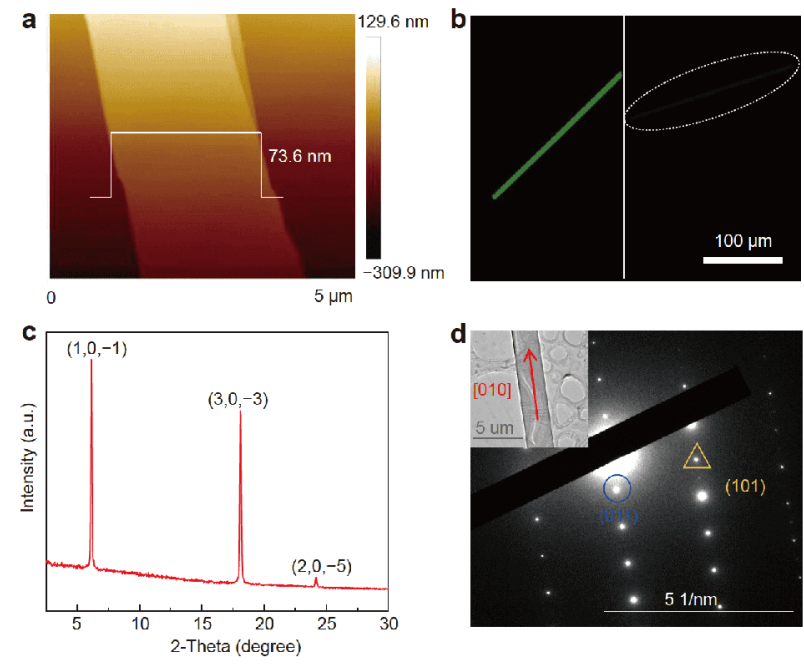

Figure 2 (a) AFM image and (b) polarizing microscope image of an individual CHICZ single crystal. (c) XRD pattern of large-area CHICZ single crystals directly grown on the OTS-modified $\mathrm{SiO}_{2} / \mathrm{Si}$ substrate. (d) TEM image of an individual CHICZ crystal and its corresponding SAED pattern shown in inside.

neighboring molecules (Fig. 1c, e) with the strong molecular packing along the $\pi-\pi$ stacking direction. The strong $\mathrm{Cl} \cdots \mathrm{C}$ interactions with the distance of $3.416 \AA$ between the neighboring CHICZ molecules are shown in Fig. 1d. And two columns of pseudo-herringbone packing form a face-to-face motif with the intermolecular distance about $3.45 \AA$ (Fig. 1e).

The CHICZ single crystals obtained by PVT and its optical microscopies are exhibited in Fig. S1. The obtained crystal has a regular rectangular shape with smooth crystal surface and around $73.6 \mathrm{~nm}$ thickness, as estimated by the atomic force microscopy (AFM, Fig. 2a), which is important for efficient charge transport in devices. Polarized microscopy shows an obvious extinction under a $45^{\circ}$ of polarizer, which demonstrates a high order alignment in the CHICZ single crystal (Fig. 2b). The X-ray diffraction (XRD) pattern of CHICZ shows sharp peaks with high intensity in Fig. $2 c$, and the baseline is relatively flat, indicating the high crystallinity of CHICZ. Combining the single crystal data of CHICZ, we infer that the main XRD diffraction peaks are assigned to the multiple $(h 0-1)$ diffractions, indicating that the $(h 0-1)$ planes are parallel to the substrate, where the $\pi-\pi$ stacking direction ( $b$ axis) is in parallel to the substrate, which is favorable for efficient charge transport in the planar phototransistors. The typical transmission electron microscopy (TEM) and its corresponding selected-area electron diffraction (SAED) pattern are shown in Fig. 2d, respectively. From the pattern, the spots in yellow triangle (101) and blue circle (011) correspond to the $d$-spacing of about 9.54 and $4.34 \AA$, which are in a good agreement with the cell parameters of the CHICZ monoclinic crystal structure. The ribbon-shape crystal of $\mathrm{CHICZ}$ are growing along the $[010]$ axis $(\pi-\pi$ stacking of $\mathrm{CHICZ}$ molecules), which is consistent with the result of XRD pattern.

Bottom-gate top-contact CHICZ single crystal phototransistors were constructed on the OTS-modified $\mathrm{SiO}_{2} / \mathrm{Si}$ substrate. A typical CHICZ single crystal based device is shown in Fig. 3a. Representative output and transfer characteristics of the device are exhibited in Fig. S2a and S2b. The devices exhibit typical p-type field-effect transporting property. From the transfer characteristics in the saturation region, we calculated the charge transfer mobility through linear fitting the curve of $\left(-I_{\mathrm{DS}}\right)^{1 / 2}$ versus $V_{\mathrm{G}}$. The maximum field-effect mobility is estimated around $0.48 \mathrm{~cm}^{2} \mathrm{~V}^{-1} \mathrm{~s}^{-1}$. The corresponding threshold voltage of the device is about $5 \mathrm{~V}$. The current on/off ratio could be up to $5.0 \times 10^{6}$. The devices have high $I_{\text {on }} / I_{\text {off }}$ and low threshold voltage (shown in Fig. S3a) due to the good energy level alignment and the high quality interface contact between CHICZ crystals and the gold electrodes.

We tested 30 devices and summarized the distribution of the mobility to assess the reproducibility of CHICZ. The relevant bar chart (Fig. S3b) shows an average mobility about $0.26 \mathrm{~cm}^{2} \mathrm{~V}^{-1} \mathrm{~s}^{-1}$. In addition, the negligible gate voltage dependence on the charge transport indicates a narrow density of state with few shallow traps in crystal state (Fig. S2c) [24], indicating the potential applications of CHICZ single crystal organic field-effect transistors in functional devices.

The light response characteristics of $\mathrm{CHICZ}$ single crystal phototransistors are shown in Fig. 3b-d. The device exhibits strong photoresponse under light irradiation. Similar to OFETs where $V_{\mathrm{G}}$ is used to control $I_{\mathrm{DS}}$, we use light instead of $V_{\mathrm{G}}$ as an independent variable to control the output (Fig. 3b) and transfer (Fig. 3c) characteristic of the phototransistors [25]. Under the light illumination, excitons dissociate into holes and electrons and greatly contribute to the increase of the device current. Specifically, the onset voltage $\left(V_{\text {on }}\right)$ moves a lot along the positive $V_{\mathrm{G}}$ with the increase of the light intensity in Fig. 3c. One of several crucial parameters evaluating the performance of phototransistors is photoresponsivity [26,27], which is defined as $R=\Delta I_{\mathrm{DS}} p^{-1} \mathrm{~A}^{-1}$, where $\Delta I_{\mathrm{DS}}=I_{\mathrm{ph}}-I_{\text {dark }}, p$ is the illumination intensity and $A$ refers to the effective area of the channel region exposed to illumination. Another crucial para- 

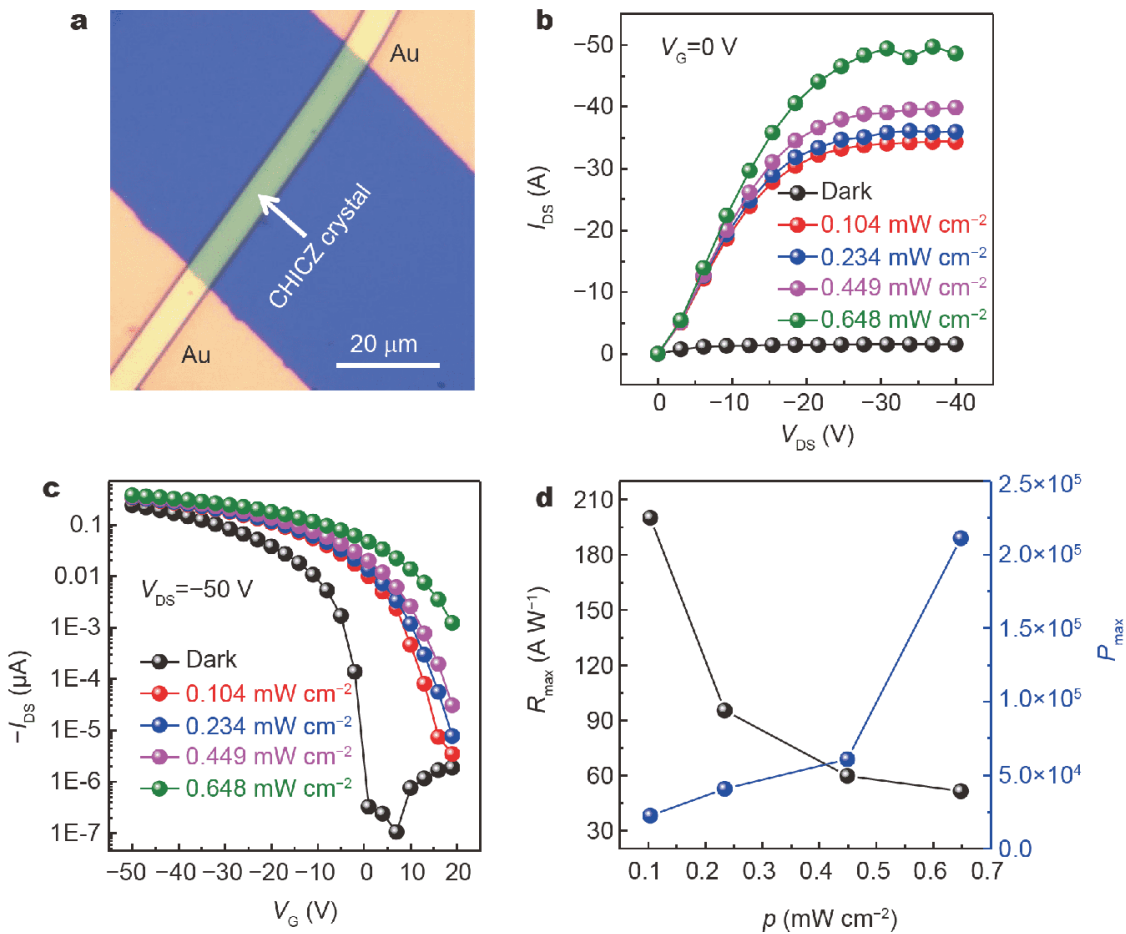

Figure 3 (a) Optical image of a typical device based on an individual CHICZ single crystal. Output (b) and transfer (c) characteristics of the phototransistor under the different light power. (d) The dependence of $R_{\max }$ and $P_{\max }$ with the intensity of light, respectively.

meter, photosensitivity, is defined as $P=\Delta I_{\mathrm{DS}} / I_{\mathrm{dark}}$. If the shot noise from the dark current is dominant [28], the specific detectivity can be expressed as $D^{*}=R /\left(2 q J_{\mathrm{d}}\right)^{1 / 2}$, where $R$ is the responsivity, $q$ is the absolute value of the electron charge $\left(1.6 \times 10^{-19}\right.$ Coulombs), and $J_{\mathrm{d}}$ is the dark current density. When $V_{\mathrm{G}}$ was kept at $1 \mathrm{~V}$ and $V_{\mathrm{DS}}$ at $-50 \mathrm{~V}$, it corresponded to a photoresponsivity of $300 \mathrm{~A} \mathrm{~W}^{-1}$ at an illumination intensity of $0.104 \mathrm{~mW} \mathrm{~cm}^{-2}$. A high photosensitivity of $2.1 \times 10^{5}$ at the $0.648 \mathrm{~mW} \mathrm{~cm}^{-2}$ is calculated under white light, so far, which is one of the highest values reported for organic materials, indicating the potential applications of CHICZ phototransistors in the field of low-power organic optoelectronics [29]. We also provide the charge transfer mechanism and energy band diagram of CHICZ under dark and illumination condition to well understand the working process of OPT, as shown in Fig. S4. In the dark operation for CHICZ-OPTs with unipolar p-channel characteristics, some of charge carriers (holes) may be trapped at the interface between the active layer and dielectric layer, leading to the relatively large threshold voltage. Under illumination, the photogenerated excitons (pairs of electrons and holes) in the conductive channel accumulate, separate and transport, which lead to the band bending in the semiconductor, the positive shift of threshold voltage and the increase of source-drain photocurrent.

In addition, we also explored the effects of light wavelength on the devices. The relationship between CHICZ performance and the wavelengths of irradiation $(\lambda=400-600 \mathrm{~nm}$, interval $10 \mathrm{~nm})$ and a fixed power of $0.02 \mathrm{~mW} \mathrm{~cm}^{-2}$ were systematically studied. Fig. 4a demonstrates the transfer characteristics of CHICZ operating under different monochromatic lights and dark state and $V_{\mathrm{DS}}=-50 \mathrm{~V}$. Compared with the curve under the dark state, an apparent increasing of $I_{\mathrm{DS}}$ with $V_{\text {th }}$ positively shifting was observed due to the generation of photo-carriers [30]. The photosensitivity was further characterized by the photo-to-dark-current ratio $\left(I_{\mathrm{ph}} /\right.$ $\left.I_{\text {dark }}\right)$, and the device under the monochromatic light of $440 \mathrm{~nm}$ showed the highest photosensitivity of $9 \times 10^{3}$, while the $I_{\mathrm{ph}} / I_{\text {dark }}$ ratio of the devices under the illumination of other light wavelengths $(490-600 \mathrm{~nm})$ was lower than 200. The wavelength dependent $I_{\mathrm{DS}}$ at $V_{\mathrm{G}}=$ $2 \mathrm{~V}$ is illustrated in Fig. $4 \mathrm{~b}$, which is consistent with the UV-vis absorption spectrum of the CHICZ single crystals in principle. Taking $400 \mathrm{~nm}$ UV light as an example, the highest photoresponsivity $\left(R_{\max }\right)$ reached $884 \mathrm{~A} \mathrm{~W}^{-1}$ when $V_{\mathrm{G}}=-14 \mathrm{~V}$. The highest photosensitivity $\left(P_{\max }\right)$ is as high as 6,390 (Fig. 4c) and the highest detectivity is 

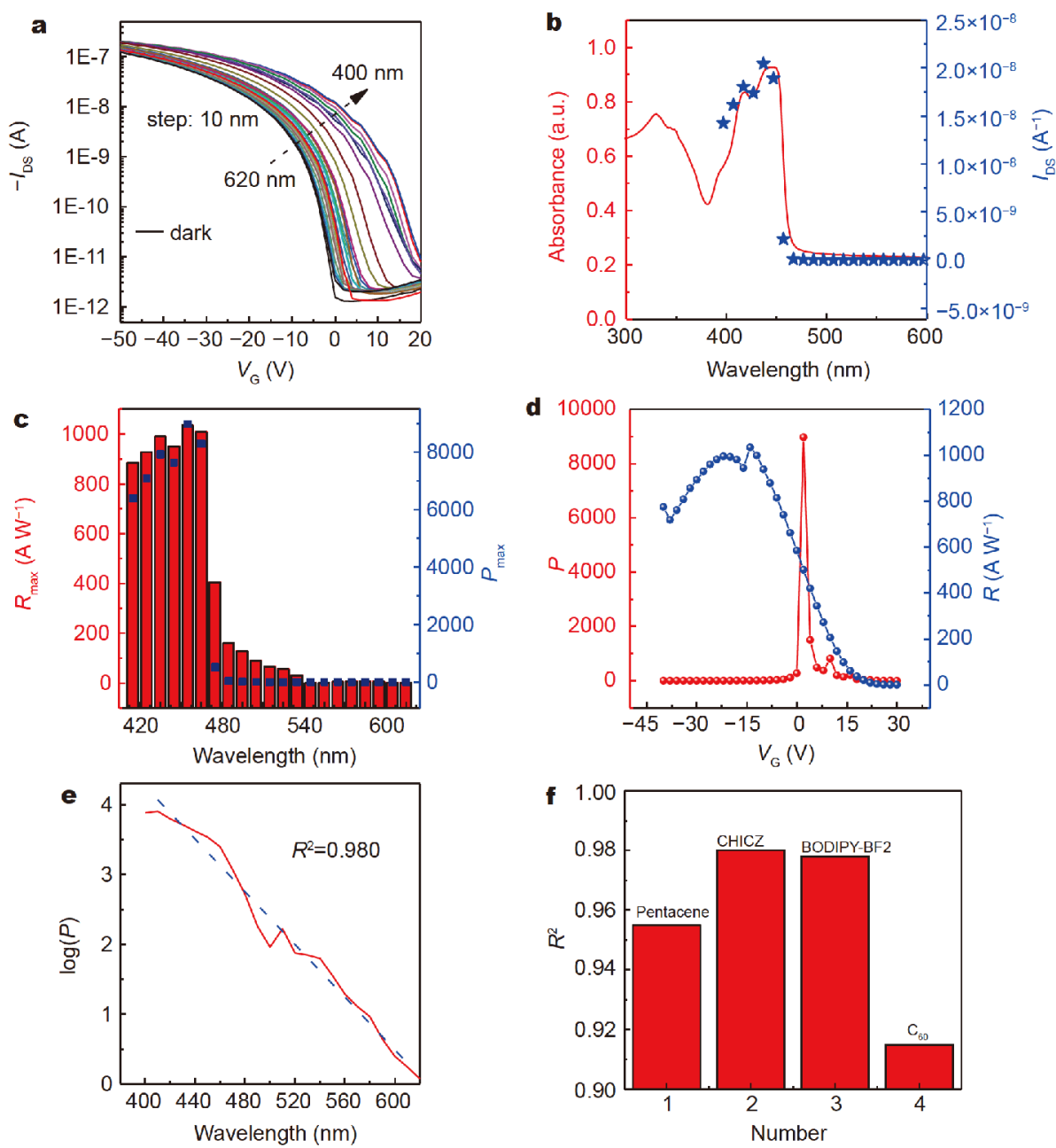

Figure 4 (a) Transfer characteristics of CHICZ phototransistor measured under dark and a series of monochromatic light illumination $(\lambda=$ 400-600 nm, interval $10 \mathrm{~nm}$ ). (b) Comparison of absorption spectrum and wavelength dependence of drain-source current for CHICZ device. (c) Wavelength dependence of maximum photoresponsivity $\left(R_{\max }\right)$ and photosensitivity $\left(P_{\max }\right)$. (d) Gate dependence of photoresponsivity and photosensitivity. (e) Wavelength dependence of $\log (P)$ based on CHICZ single crystals. (f) The linear correlation comparison of several materials.

$5.9 \times 10^{14}$ Jones under the condition of $V_{\mathrm{G}}=2 \mathrm{~V}$. This is one of the best organic phototransistors performances towards UV lights [31-34]. Fig. 4c illustrates the optoelectronic characteristics of CHICZ-based phototransistors in the region of UV-vis wavelength, demonstrating the wide sensitivity and selectivity of the CHICZbased phototransistors. Under the visible light irradiation of $500 \mathrm{~nm}, R_{\max }$ and $P_{\max }$ are only $66 \mathrm{~A} \mathrm{~W}^{-1}$ (at $V_{\mathrm{G}}=$ $-28 \mathrm{~V}$ ) and $8\left(\right.$ at $V_{\mathrm{G}}=2 \mathrm{~V}$ ), respectively, corresponding to $7 / 100$ and $1 / 1,000$ of the value obtained under the irradiation of $400 \mathrm{~nm}$. Fig. $4 \mathrm{~d}$ shows the $R$ and $P$ as the $V_{\mathrm{G}}$ gradually increased under the illumination of $400 \mathrm{~nm}$ light and the light intensity is $0.02 \mathrm{~mW} \mathrm{~cm}^{-2}$, and the $R_{\max }$ and $P_{\max }$ appear at around -15 and $0 \mathrm{~V}$, respectively.

Furthermore, when we further analyzed the data of $P_{\max }$ with $\log (P)$ versus light wavelength, an interesting linear relationship between the $\log (P)$ and light wavelength was observed with the defined formula of $y=0.019 x+11.606$, where the linear correlation coefficient $R^{2}=0.98$ (as shown in Fig. 4e), indicating a linear correlation. Such linear relationship will be helpful for organic photodetectors to be used in the unique wavelength detection. Moreover, we systematically summarized the data of organic phototransistors reported in literatures regarding their wavelength dependence in Table S1. Interestingly, through the similar analysis process, most of the devices demonstrate nearly linear relationship between $\log (P)$ versus wavelength (Fig. S5). Amongst them, CHICZbased single crystal phototransistors exhibit the highest linear correlation coefficient, which is ascribed to the 
absorption ability, charge transport property as well as the photoelectric conversion of organic materials.

\section{CONCLUSIONS}

In summary, high performance CHICZ single crystalbased phototransistors were constructed. The devices exhibited high optoelectronic performances with photoresponsivity of $3 \times 10^{3} \mathrm{~W} \mathrm{~cm}^{-2}$, photosensitivity of $2 \times 10^{4}$ and detectivity $D^{\star}$ of up to $8.4 \times 10^{14}$ Jones. In addition, we also tested a series of light responses at the same light intensity with different wavelength for CHICZ-based OPTs. Interestingly, except for the typical wavelength responsetivity, the device also demonstrated good linear relationship for $\log (P)$ versus the light wavelength with the linear correlation coefficient $\left(R^{2}\right)$ of 0.98 , indicating its unique wavelength detection in optoelectronic devices.

Received 11 September 2018; accepted 19 October 2018; published online 15 November 2018

$1 \mathrm{Gu} \mathrm{P}$, Yao Y, Feng $\mathrm{L}$, et al. Recent advances in polymer phototransistors. Polym Chem, 2015, 6: 7933-7944

2 Guo Y, Yu G, Liu Y. Functional organic field-effect transistors. Adv Mater, 2010, 22: 4427-4447

3 Wang $\mathrm{H}$, Liu $\mathrm{H}$, Zhao Q, et al. Three-component integrated ultrathin organic photosensors for plastic optoelectronics. Adv Mater, 2016, 28: 624-630

4 Zhao G, Liu J, Meng Q, et al. High-performance UV-sensitive organic phototransistors based on benzo[1,2-b:4,5-b] dithiophene dimers linked with unsaturated bonds. Adv Electron Mater, 2015, 1: 1500071

$5 \mathrm{Hu}, \mathrm{W}$. Organic Optoelectronics. Weinheim: Wiley-VCH, 2013

6 Sekitani $\mathrm{T}$, Zschieschang $\mathrm{U}$, Klauk $\mathrm{H}$, et al. Flexible organic transistors and circuits with extreme bending stability. Nat Mater, 2010, 9: 1015-1022

7 Zhou B, Zhou J, Chen Y, et al. Performance improvement of organic phototransistors by using polystyrene microspheres. Sci China Mater, 2018, 61: 737-744

8 Li Q, Ding S, Zhu W, et al. Recent advances in one-dimensional organic $\mathrm{p}-\mathrm{n}$ heterojunctions for optoelectronic device applications. J Mater Chem C, 2016, 4: 9388-9398

9 Wang C, Ren X, Xu C, et al. N-type 2D organic single crystals for high-performance organic field-effect transistors and near-infrared phototransistors. Adv Mater, 2018, 30: 1706260

10 Qin X, Dong H, Hu W. Green light-emitting diode from bromine based organic-inorganic halide perovskite. Sci China Mater, 2015, 58: $186-191$

11 Wang C, Dong H, Jiang L, et al. Organic semiconductor crystals. Chem Soc Rev, 2018, 47: 422-500

12 Dong $\mathrm{H}$, Yan $\mathrm{Q}, \mathrm{Hu} \mathrm{W}$, et al. Multilevel investigation of charge transport in conjugated polymers-new opportunities in polymer electronics. Acta Polym Sin, 2017, 8: 1246-1260

13 Kim D, Lee D, Lee $\mathrm{H}$, et al. High-mobility organic transistors based on single-crystalline microribbons of triisopropylsilylethynyl pentacene via solution-phase self-assembly. Adv Mater, 2007, 19: 678-682

14 Reese C, Bao Z. Organic single-crystal field-effect transistors.
Mater Today, 2007, 10: 20-27

15 Li Q, Liu S, Chen H, et al. Alignment and patterning of organic single crystals for field-effect transistors. Chin Chem Lett, 2016, 27: $1421-1428$

$16 \mathrm{Wu} \mathrm{J}, \mathrm{Li} \mathrm{Q}$, Xue G, et al. Preparation of single-crystalline heterojunctions for organic electronics. Adv Mater, 2017, 29: 1606101

17 Yang F, Cheng S, Zhang X, et al. 2D organic materials for optoelectronic applications. Adv Mater, 2018, 30: 1702415

18 Zhou K, Chen H, Dong H, et al. Comparable charge transport property based on S...S interactions with that of $\pi-\pi$ stacking in a bis-fused tetrathiafulvalene compound. Sci China Chem, 2017, 60: 510-515

19 Gu P, Yao Y, Dong H, et al. Preparation, characterization and field effect transistor applications of conjugated polymer micro/nanocrystal. Acta Polym Sin, 2014, 30: 1029-1040

20 Zhao G, Dong H, Jiang L, et al. Single crystal field-effect transistors containing a pentacene analogue and their application in ethanol vapor detection. Appl Phys Lett, 2012, 101: 103302

21 Laudise RA, Bridenbaugh PM, Kloc C, et al. Organo-thermal crystal growth of $a 6$ thiophene. J Cryst Growth, 1997, 178: 585-592

22 Jiang L, Gao J, Wang E, et al. Organic single-crystalline ribbons of a rigid " $\mathrm{H}$ "-type anthracene derivative and high-performance, short-channel field-effect transistors of individual micro/nanometer-sized ribbons fabricated by an "organic ribbon mask" technique. Adv Mater, 2008, 20: 2735-2740

23 Zhao G, Dong H, Zhao H, et al. Substitution effect on molecular packing and transistor performance of indolo[3,2-b]carbazole derivatives. J Mater Chem, 2012, 22: 4409-4417

$24 \mathrm{Li} \mathrm{J}$, Zhou K, Liu J, et al. Aromatic extension at 2,6-positions of anthracene toward an elegant strategy for organic semiconductors with efficient charge transport and strong solid state emission. J Am Chem Soc, 2017, 139: 17261-17264

25 Dong $\mathrm{H}, \mathrm{Bo} \mathrm{Z}, \mathrm{Hu} \mathrm{W}$. High performance phototransistors of a planar conjugated copolymer. Macromol Rapid Commun, 2011, 32: 649-653

26 Dong $\mathrm{H}, \mathrm{Li} \mathrm{H}$, Wang E, et al. Phototransistors of a rigid rod conjugated polymer. J Phys Chem C, 2008, 112: 19690-19693

27 Yang X, Li Q, Hu G, et al. Controlled synthesis of high-quality crystals of monolayer $\mathrm{MoS}_{2}$ for nanoelectronic device application. Sci China Mater, 2016, 59: 182-190

28 Gong X, Tong M, Xia Y, et al. High-detectivity polymer photodetectors with spectral response from $300 \mathrm{~nm}$ to $1450 \mathrm{~nm}$. Science, 2009, 325: 1665-1667

29 Wang C, Liu Y, Wei Z, et al. Biphase micro/nanometer sized single crystals of organic semiconductors: control synthesis and their strong phase dependent optoelectronic properties. Appl Phys Lett, 2010, 96: 143302

30 Kakinuma T, Kojima H, Kawamoto T, et al. Giant phototransistor response in dithienyltetrathiafulvalene derivatives. J Mater Chem C, 2013, 1: 2900-2905

31 Baeg KJ, Binda $\mathrm{M}$, Natali $\mathrm{D}$, et al. Organic light detectors: photodiodes and phototransistors. Adv Mater, 2013, 25: 4267-4295

32 Noh YY, Kim DY, Yoshida Y, et al. High-photosensitivity pchannel organic phototransistors based on a biphenyl end-capped fused bithiophene oligomer. Appl Phys Lett, 2005, 86: 043501

33 Noh YY, Ghim J, Kang SJ, et al. Effect of light irradiation on the characteristics of organic field-effect transistors. J Appl Phys, 2006, 100: 094501

34 Lucas B, El Amrani A, Chakaroun M, et al. Ultraviolet light effect 
on electrical properties of a flexible organic thin film transistor. Thin Solid Films, 2009, 517: 6280-6282

Acknowledgements The authors acknowledge financial support from the Ministry of Science and Technology of China (2017YFA0204503 and 2016YFB0401100), the National Natural Science Foundation of China (51725304, 51633006, 51703159 and 51733004), and the Strategic Priority Research Program (XDB12030300) of the Chinese Academy of Sciences.

Author contributions Dong $\mathrm{H}$ and $\mathrm{Hu} \mathrm{W}$ conceived and designed the experiments. Zhou $\mathrm{K}$ provided the help for constructing micro/nanosingle crystal transistors and electrical measurement. Zhang Q helped paint. Liu D, Liu J, Li J and Zhao Q helped analyze the experimental data. Dong H, Hu M, and Liu J co-wrote the manuscript and all the authors contributed to the general discussion.

Conflict of interest The authors declare that they have no conflict of interest.

Supplementary information The supporting data are available in the online version of the paper.

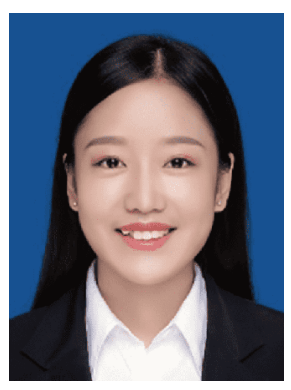

Mengxiao Hu obtained her BSc degree from Qingdao University in 2016. Now she is a master student at the Department of Chemistry, Capital Normal University (CNU), China. Her current research focuses on organic optoelectronic functional devices.

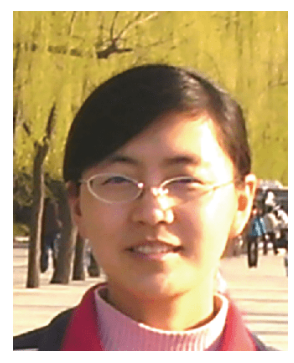

Huanli Dong is a Professor of the Institute of Chemistry, Chinese Academy of Sciences (CAS). She received her PhD degree from the Institute in 2009 after she got her MSc degree from Fujian Institute of Research on the Structure of Material, CAS, in 2006. Her current research focuses on organic/polymer optoelectronic materials and devices, including molecular structure design, solid state structure modulation as well as device physics.

\section{具有独特波长检测特性的有机单晶光电晶体管}

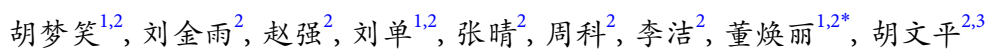

摘要 高质量的 2,8 -二氯 $-5,11$-二已基-吲哚 $[3,2-b]$ 咔唑(CHICZ) 单晶被应用于光电晶体管, 并表现出高性能的光响应度 $\left(R_{\max }=3 \times 10^{3} \mathrm{~A} \mathrm{~W}^{-1}\right)$ 和光敏感性 $\left(P_{\max }=2 \times 10^{4}\right.$ 以及 $\left.D_{\text {max }}{ }^{*}=8.4 \times 10^{14} \mathrm{Jones}\right)$. 此外, 我们首次发现当这些器件被一系列相同强度但不同波长的 光照亮时, 光敏度的对数与波长呈现非常好的线性关系. 这一现象为拓展有机光控晶体管在波长检测中的应用提供了新视角. 